\title{
A novel microdeletion syndrome involving 5q14.3-q15: clinical and molecular cytogenetic characterization of three patients
}

Hartmut Engels ${ }^{*}{ }^{1}$, Eva Wohlleber ${ }^{1}$, Alexander Zink ${ }^{1}$, Juliane Hoyer ${ }^{2}$, Kerstin U Ludwig ${ }^{3}$, Felix F Brockschmidt ${ }^{3}$, Dagmar Wieczorek ${ }^{4}$, Ute Moog ${ }^{5}$, Birgit Hellmann-Mersch ${ }^{6}$, Ruthild G Weber ${ }^{1}$, Lionel Willatt ${ }^{7}$, Martina Kreiß-Nachtsheim ${ }^{1}$, Helen V Firth ${ }^{7}$ and Anita Rauch ${ }^{2}$

${ }^{1}$ Institute of Human Genetics, Rheinische Friedrich-Wilhelms-University, Bonn, Germany; ${ }^{2}$ Institute of Human Genetics, Friedrich-Alexander University Erlangen-Nuremberg, Erlangen, Germany; ${ }^{3}$ Department of Genomics, Life and Brain Center, University of Bonn, Bonn, Germany; ${ }^{4}$ Institut für Humangenetik, Universitätsklinikum Essen, Universität Duisburg-Essen, Essen, Germany; ${ }^{5}$ Institute of Human Genetics, Ruprecht-Karls-University, Heidelberg, Germany; ${ }^{6}$ Department of Child Neurology and Developmental Pediatrics, LVR-Klinikum Bonn, Bonn, Germany; ${ }^{7}$ Department of Medical Genetics, Addenbrooke's Hospital NHS Trust, Cambridge, UK

Molecular karyotyping is being increasingly applied to delineate novel disease causing microaberrations and related syndromes in patients with mental retardation of unknown aetiology. We report on three unrelated patients with overlapping de novo interstitial microdeletions involving 5q14.3-q15. All three patients presented with severe psychomotor retardation, epilepsy or febrile seizures, muscular hypotonia and variable brain and minor anomalies. Molecular karyotyping revealed three overlapping microdeletions measuring 5.7, 3.9 and $3.6 \mathrm{Mb}$, respectively. The microdeletions were identified using single nucleotide polymorphism (SNP) arrays (Affymetrix $100 \mathrm{~K}$ and Illumina 550K) and array comparative genomic hybridization ( $1 \mathrm{Mb}$ Sanger array-CGH). Confirmation and segregation studies were performed using fluorescence in situ hybridization (FISH) and quantitative PCR. All three aberrations were confirmed and proven to have occurred de novo. The boundaries and sizes of the deletions in the three patients were different, but an overlapping region of around $1.6 \mathrm{Mb}$ in $5 q 14.3$ was defined. It included five genes: CETN3, AC093510.2, POLR3G, LYSMD3 and the proximal part of GPR98/MASS1, a known epilepsy gene.

Haploinsufficiency of GPR98/MASS1 is probably responsible for the seizure phenotype in our patients. At least one other gene contained in the commonly deleted region, LYSMD3, shows a high level of central nervous expression during embryogenesis and is also, therefore, a good candidate gene for other central nervous system (CNS) symptoms, such as psychomotor retardation, brain anomalies and muscular hypotonia of the 5 q14.3 microdeletion syndrome.

European Journal of Human Genetics (2009) 17, 1592-1599; doi:10.1038/ejhg.2009.90; published online 27 May 2009

Keywords: microdeletion 5q14.3; mental retardation; seizures; brain anomalies; molecular karyotyping

*Correspondence: Dr H Engels, Institute of Human Genetics, University of Bonn, Wilhelmstraße 31, D-53111 Bonn, Germany.

Tel: + 490228287 22371; Fax: + 490228287 22380;

E-mail: hartmut.engels@ukb.uni-bonn.de

Received 30 October 2008; revised 4 February 2009; accepted 8 April 2009; published online 27 May 2009
Introduction

Molecular karyotyping by single nucleotide polymorphism (SNP) arrays and array-CGH allows high-resolution screening of the whole human genome for DNA copy number changes. When applied to cohorts of patients with mental 
retardation (MR) and dysmorphic features, this approach detects causative microdeletions and microduplications in $10-15 \%$ of the patients, most of which are scattered across the genome. ${ }^{1-3}$ For newly described copy number changes, de novo origin versus inheritance from an unaffected parent is used to help to differentiate between causative copy number changes and benign variants that affect more than $12 \%$ of the human genome. ${ }^{4}$ Causative familial copy number changes with reduced penetrance have also been described, however. $^{2,5,6}$ Several patients with similar copy number changes and a similar clinical presentation are, therefore, needed to allow reliable genotype-phenotype correlation. Recently, some recurrent rearrangements have been described for, amongst others, chromosomal regions $1 \mathrm{q} 21.1,{ }^{5} 1 \mathrm{q} 41 \mathrm{q} 42,{ }^{7}$ $2 \mathrm{p} 15 \mathrm{p} 16.1,^{8} 16 \mathrm{p} 13.11,{ }^{9,10} 18 \mathrm{q} 21^{11-13}$ and $17 \mathrm{q} 21.31 .^{14-16}$

Here, we report on three unrelated patients with overlapping microdeletions in chromosomal region 5q14.3q15. After diagnosis of patient 3, a 'call for patients' within the German MRNET yielded patient 1. Patient 2 was then ascertained through the DECIPHER database (https:// decipher.sanger.ac.uk). Clinical similarities between all three patients include severe MR, epilepsy and/or febrile seizures, and muscular hypotonia. In addition to the clinical characterization of the new syndrome, we delineate a critical region, presumed to contain haploinsufficient genes, that is involved in the aetiology of the observed overlapping phenotypic features.

\section{Case reports}

Ethical approval for this study was obtained from the ethics committees of all participating centres and informed consent for genetic investigations into mental retardation was obtained from participating families.

\section{Patient 1}

The female proband was the second of three children born to a healthy non-consanguineous couple of Turkish origin. The girl was born 11 days postterm following an uneventful pregnancy. At birth, the umbilical cord was wrapped around the neck. Her birthweight was 3420 g (25-50th centile/-0.37 SDS), length was $53 \mathrm{~cm}(50-75 \mathrm{th}$ centile/ +0.36 SDS) and OFC was $38 \mathrm{~cm}(>97 \mathrm{th}$ centile/ +2.15 SDS). Apgar scores were 9, 9, 10 and cord $\mathrm{pH}$ was 7.27. Nine hours after birth, the child was referred to the paediatric intensive care ward with muscular hypotonia and tachydyspnoea, where she subsequently developed infantile spasms. Cerebral MRI studies showed aplasia of the cerebellar vermis and posterior part of the corpus callosum, multiple plexus cysts and enlarged occipital horns of the lateral ventricles. Chromosome studies, screening for perinatal infections and metabolic disorders and ophthalmological investigation revealed no abnormalities. Extensive EEG studies at the age of 4 months showed hypsarrhythmia, which was unresponsive to ACTH therapy but a combination of ACTH and vigabatrin reduced the frequency of myoclonic seizures although rolling eye movements persisted. Echocardiography showed concentric myocardial hypertrophy (ventricular septum $6-7 \mathrm{~mm}$, left ventricular posterior wall $6 \mathrm{~mm}$ ). X-ray studies showed incomplete closure of thoracic vertebral arches Th2-Th10. Chronic constipation and increased sweating was noted. The child showed no psychomotor development. Due to the clinical suspicion of Aicardi syndrome, ophthalmological investigation was repeated at the age of 3 years, but revealed only simple bilateral optic atrophy. At the age of 6 years, the girl was without anticonvulsant medication and presented with occasional seizures, truncal hypotonia and bilateral pes equines. Her psychomotor development was severely retarded and still comparable to that of a neonate. She was fed with pureed food only, had normal measurements (length: $111 \mathrm{~cm}$ (10th centile/-1.1 SDS), OFC: $52.5 \mathrm{~cm}$ (90-97th centile/ +1.1 SDS)) and suffered from frequent upper respiratory tract infections. She showed no facial dysmorphism, but her ears were relatively large and her eyebrows were relatively broad. She had bilateral transverse palmar creases, short halluces and three café-au-lait spots (one spot of $0.5 \times 1.5 \mathrm{~cm}$ on the back, two spots of $2 \times 3 \mathrm{~cm}$ in the inguinal region). This patient was mentioned within a larger series previously (patient 77). ${ }^{3}$

\section{Patient 2}

Patient 2 was born following a pregnancy in which a markedly increased nuchal translucency measurement had been noted. A CVS revealed a normal female karyotype and fetal anomaly ultrasound imaging was normal. She was born at 40 weeks gestation weighing $3300 \mathrm{~g}$ (25-50th centile/-0.41 SDS) with Apgar scores of $8,{ }^{1} 9 .{ }^{5}$ There were severe feeding difficulties postnatally with a poor suckling ability and frequent vomiting and she failed to thrive. Her development was very delayed with a social smile from 5 months and truncal hypotonia. When reviewed at the age of 7 years she was attending a school for children with special educational needs. She was not yet able to sit independently but had recently begun to babble. Her overall developmental level was equivalent to a child of approximately 6 months. She had a history of several febrile seizures and was maintained on anticonvulsants although she had been seizure free for the previous 3 years. She had a striking visual preoccupation with stripes. She had marked brachycephaly with a low anterior hairline. Facial features included downslanting palpebral fissures and a philtral haemangioma, which had been present since birth (Figure 1f and g). Her growth parameters at the age of 8 years were as follows: height, $120.4 \mathrm{~cm}$ (9th centile/-1.58 SDS); weight, $21.3 \mathrm{~kg}$ (2nd-9th centile/BMI 14.7); OFC, $51.3 \mathrm{~cm}$ (2nd-9th centile/-0.38 SDS).

A cranial MRI scan in infancy showed prominence of the arachnoid spaces in the perivascular areas. An EEG showed 


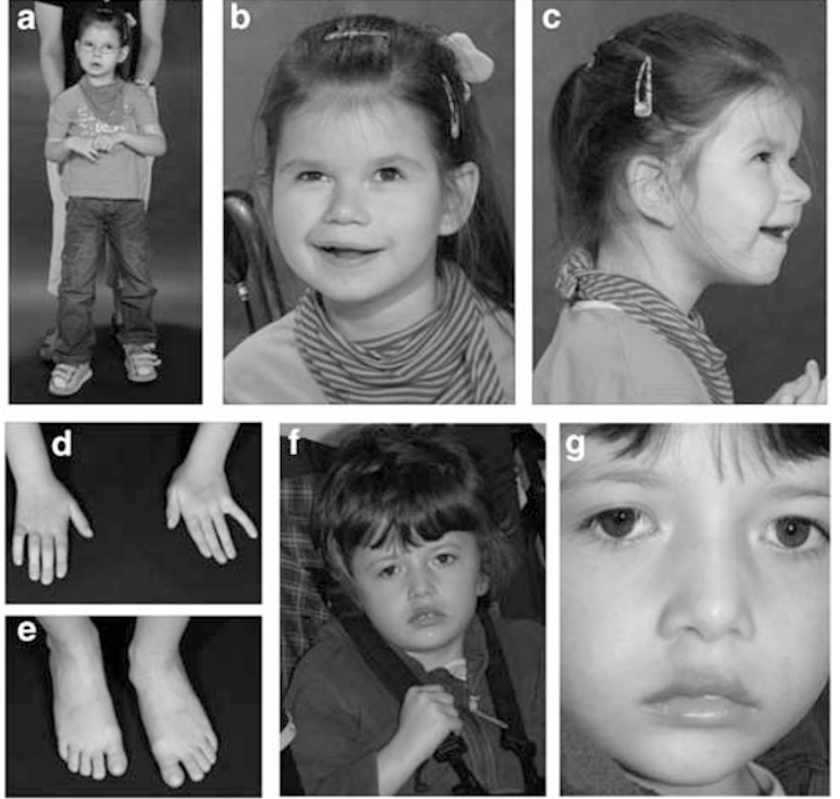

Figure 1 Variable mild dysmorphisms of patients 2 and 3. (a-e) Patient 3 at the age of 7 years showing minor anomalies, such as relatively large ears, and long and slender fingers. The seemingly narrowed right palpebral fissure is due to the patient's facial expression and thus not representative. (f, g) Patient 2 at the age of 5 years showing mildly downslanting palpebral fissures, downturned corners of the mouth and philtral haemangioma. Consent to the publication of clinical pictures of patient 1 could not be obtained.

high amplitude prominent rhythmical activity in the temporal regions with generalized bursts of spike and slow waves accompanied by some myoclonic jerking of the arms.

\section{Patient 3}

Patient 3 is a girl, the third-born child of non-consanguineous German parents with an unremarkable family history. After an uneventful pregnancy, she was born at term with normal measurements (weight: $3510 \mathrm{~g}$ (50th centile/+0.09 SDS), length: $51 \mathrm{~cm}$ (25th-50th centile/ -0.32 SDS), OFC: $35 \mathrm{~cm}$ (50th centile/+0.08 SDS)). Apgar scores were $9,9,10$. No malformations or other abnormalities were apparent. According to her mother, she slept much more than her healthy brothers had done during the first months of life and she was very sensitive to noise. At the age of 4 months, psychomotor delay and severe muscular hypotonia were noted by a paediatrician. Her developmental milestones were delayed (head control: later than 1 year of age, unsupported sitting approximately at 2.5 years of age, no unsupported walking at the age of 7 years). She began uttering syllables (mamama, papapa) at the age of 3 years. At the age of 6 years, she had learned to say 'mama' in a directed fashion. Her language comprehension was reported as being better, and she could express basic needs using an electronic speaking aid.
At the age of 5 months, hyperopia $(+5.5 \mathrm{dpt}$ at the age of 5 years) and strabismus convergens were diagnosed. An EEG at the age of 9 months revealed no anomalies. Cranial MRIs (at 6 months and 2 years 3 months) demonstrated moderate atrophy of the supra- and infratentorial region, a slightly enlarged ventricular system and unspecific leucoencephalopathy. At the age of 4 years 9 months, the first atypical absences occurred. An EEG documented short focal seizures accompanied by atypical absences. Treatment with oxacarbazepine commenced. Later, complex partial seizures were reported. At the age of 5 years 3 months, one grand mal seizure occurred.

At the time of our first evaluation at the age of 5 years 3 months, she presented with severe MR and global developmental delay. Social interactions were very limited. Formal neuropsychological testing was not possible. Neurological examination revealed muscular hypotonia predominantly of the trunk. She could neither roll from her back to her front, nor move without support, although used her wheelchair adroitly.

On our second examination at 6 years 9 months of age, only mild dysmorphisms, such as simple ears, a slightly narrowed supraorbital region and slightly upslanting palpebral fissures were noted (Figure $1 \mathrm{a}-\mathrm{e}$ ). At the time of this examination, her growth parameters were as follows: height, $115 \mathrm{~cm}$ (10th centile/-1.53 SDS); weight, $21 \mathrm{~kg}$ (25th centile); OFC, $49.5 \mathrm{~cm}$ (10th-25th centile/ -1.61 SDS). Her mouth was frequently open and she hypersalivated. Hypotonia of the pharyngeal muscles had led to feeding problems and her diet, therefore, included only fluids or pureed food. She displayed teeth grinding and stereotypic dyskinetic hand movements. Her seizure medication had been changed to a combination of oxacarbazepine and levitiracetam. An EEG showed no epileptiform activity although short absence-like seizures and tonic seizures still occurred. Echocardiography and physical examination revealed no evidence of malformations. Genetic analyses with normal results included chromosome analyses (400-500 band level), Angelman syndrome and Rett syndrome testing.

\section{Material and methods \\ Patient 1}

Molecular karyotyping using a GeneChip Human Mapping 100K SNP array (Affymetrix) was performed as previously described. ${ }^{3}$ This patient was mentioned within a larger series previously ( ${ }^{3}$ patient 77$)$. The deletion was confirmed by twocolour FISH with locus-specific probe RP11-88D22 and a subtelomeric control probe on lymphocyte metaphase spreads of the patient and her parents as described previously. ${ }^{3}$

\section{Patient 2}

BAC array analysis using the Sanger $1 \mathrm{Mb}$ array was performed as previously described. ${ }^{17}$ FISH was performed 
with multiple tile path RPCI11 clones obtained from BAC PAC resources (http://bacpac.chori.org) directly labelled with either Vysis Spectrum Orange-dUTP or Spectrum Green-dUTP (Vysis) as previously described ${ }^{17}$ to size the deletion in the proband and to screen the parents.

\section{Patient 3}

Genomic DNA of Patient 3 was analysed using an Illumina Sentrix HumanHap 550-Duo v3Beadchip. Quality control demonstrated a call rate of $99.6 \%$. Putative CNVs were identified using the QuantiSNP algorithm. ${ }^{18}$ In brief, QuantiSNP is a Hidden-Markov Model-based algorithm for high-resolution $\mathrm{CNV}$ detection that uses $\log 2 \mathrm{R}$ ratio (LRR) values (a normalized measure of the signal intensity for each SNP) and B allele frequency (BAF) values (an allelic intensity ratio for each SNP) to generate CNV calls. As a measure of confidence, a log Bayes factor is computed for each CNV. We computed LRR and BAF values with the Beadstudio v3.1.3.0 genotyping module (Illumina Inc.) and analysed these with QuantiSNP using default settings. We discarded all CNV calls with a log Bayes factor value below 7, containing less than 5 SNPs or a genomic size below $10 \mathrm{~kb}$. Putative CNVs were disregarded if they were covered entirely by known variants according to the Database of Genomic Variants (http://projects.tcag.ca/ variation/) or contained no known genes. For qPCR analyses, we designed 3 amplicons localized in the coding regions of GPR98/MASS1, CETN3 and LYSMD3. qPCR was performed on an ABI Prism 7900HT Fast Real-Time PCR System (Applied Biosystems, Darmstadt, Germany) using SYBR Green for detection. Reaction mixtures contained $0.2 \mu \mathrm{M}$ of each primer and $5 \mu \mathrm{l}$ of Power SYBR Green PCR Master Mix (Applied Biosystems). Each assay included DNA from four control persons and the patient DNA at a final concentration of $20 \mathrm{ng} / \mu \mathrm{l}$ in triplicate (total volume: $10 \mu \mathrm{l}$ ). The cycling conditions were as follows: initiation, $50^{\circ} \mathrm{C}$ for $2 \mathrm{~min}$; denaturation, $95^{\circ} \mathrm{C}$ for $10 \mathrm{~min}$, followed by 40 cycles at $95^{\circ} \mathrm{C}$ for $15 \mathrm{~s}$, and a combined annealing and extension step at $60^{\circ} \mathrm{C}$ for $60 \mathrm{~s}$. To exclude the presence of unspecific products, a melting-curve analysis of the products was performed. The threshold cycles $\left(C_{t}\right)$ values were normalized using the $C_{\mathrm{t}}$ value of three reference genes (BNC1, CFTR and RNAseP subunit p38) for each DNA sample. Relative quantification was performed by use of the comparative $C_{\mathrm{t}}$ method. Additionally, qPCR screening for the microdeletion was performed on the genomic DNA of 57 patients with MR and an overlapping clinical spectrum, for example, (febrile) seizures/pathological EEG, pathological cranial MRI, muscular hypotonia, autistic behaviour or stereotypies. FISH was performed using BlueFISH tile BAC probes RP11-12D3 and RP11-29K14 from the deleted region according to manufacturer's instructions (BlueGnome Ltd, Cambridge, United Kingdom) and as published previously. ${ }^{2}$

\section{Results \\ Patient 1}

Molecular karyotyping using a GeneChip Human Mapping 100K SNP array (Affymetrix) revealed a $5.69 \mathrm{Mb}$ deletion of 5q14.3-q15 containing 234 SNP probes (start SNP: rs10514301, genomic position/NCBI assembly 36: bp 87975410; end SNP rs9314105, bp 93668872 ; Figure 2a). FISH with BAC probe RP11-88D22 from the deleted region in patient 1 on metaphase preparations of the patient and her parents confirmed the de novo occurrence of the deletion (Figure 2d). The patient's karyotype according to ISCN 2005 is arr cgh $5 q 14.3 q 15($ rs10514301 $\rightarrow$ rs9314105) $\times 1 \mathrm{dn}$.

\section{Patient 2}

BAC array analysis using the Sanger $1 \mathrm{Mb}$ array showed a deletion of 5 clones localized in 5q14.3. (start clone: RP11-72L22, midpoint genomic position $86.4 \mathrm{Mb}$; end clone RP11-302L17, midpoint genomic position: $89.6 \mathrm{Mb}$; Figure 2c). FISH on lymphocyte metaphase preparations of the patient and her parents confirmed the de novo occurrence of the deletion and was used to define the breakpoints in the proband (Figure 2e). At the centromeric end the normal flanking clone was RP11-265O6 (bp 85851532 - bp 85988261 ) and the first deleted clone was RP11-291O24 (bp 86206067 - bp 86341427 ). At the telomeric end, the last deleted clone was RP11-62E10 (bp 90044836 - bp 90139366$)$ and the normal flanking clone was RP11-351K10 (bp 90139366 - bp 90265224 ). The deletion size determined by the distance of the genomic end points of the deleted clones RP11-291O24 and RP11-62E10 was $3.93 \mathrm{Mb}$ and the patient's karyotype is arr cgh 5q14.3(RP11-291O24 $\rightarrow$ RP11-62E10) $\times 1 \mathrm{dn}$.

\section{Patient 3}

Illumina HumanHap 550 BeadChip analysis revealed a $3.574 \mathrm{Mb}$ heterozygous deletion containing 524 SNPs in 5q14.3-q15 (start SNP: rs10223241, genomic position: bp 88,448,144; end SNP rs17664587, bp 92022455; Figure $2 \mathrm{~b}$ ). qPCR analyses of patient 3 and her parents confirmed the deletion in patient 3 and demonstrated its de novo occurrence. qPCR screening for the microdeletion performed on the genomic DNA of 57 additional patients with an overlapping clinical spectrum (eg, MR and seizures, muscular hypotonia or stereotypies) identified no additional microdeletions.

FISH with two BAC probes from the region deleted in patient 3 (RP11-12D3, RP11-29K14) performed on lymphocyte metaphase preparations of the patient and her parents confirmed the de novo occurrence of the deletion (Figure 2f). The patient's karyotype is arr cgh 5q14.3q15 $(\mathrm{rs} 10223241 \rightarrow \mathrm{rs} 17664587) \times 1 \mathrm{dn}$. 

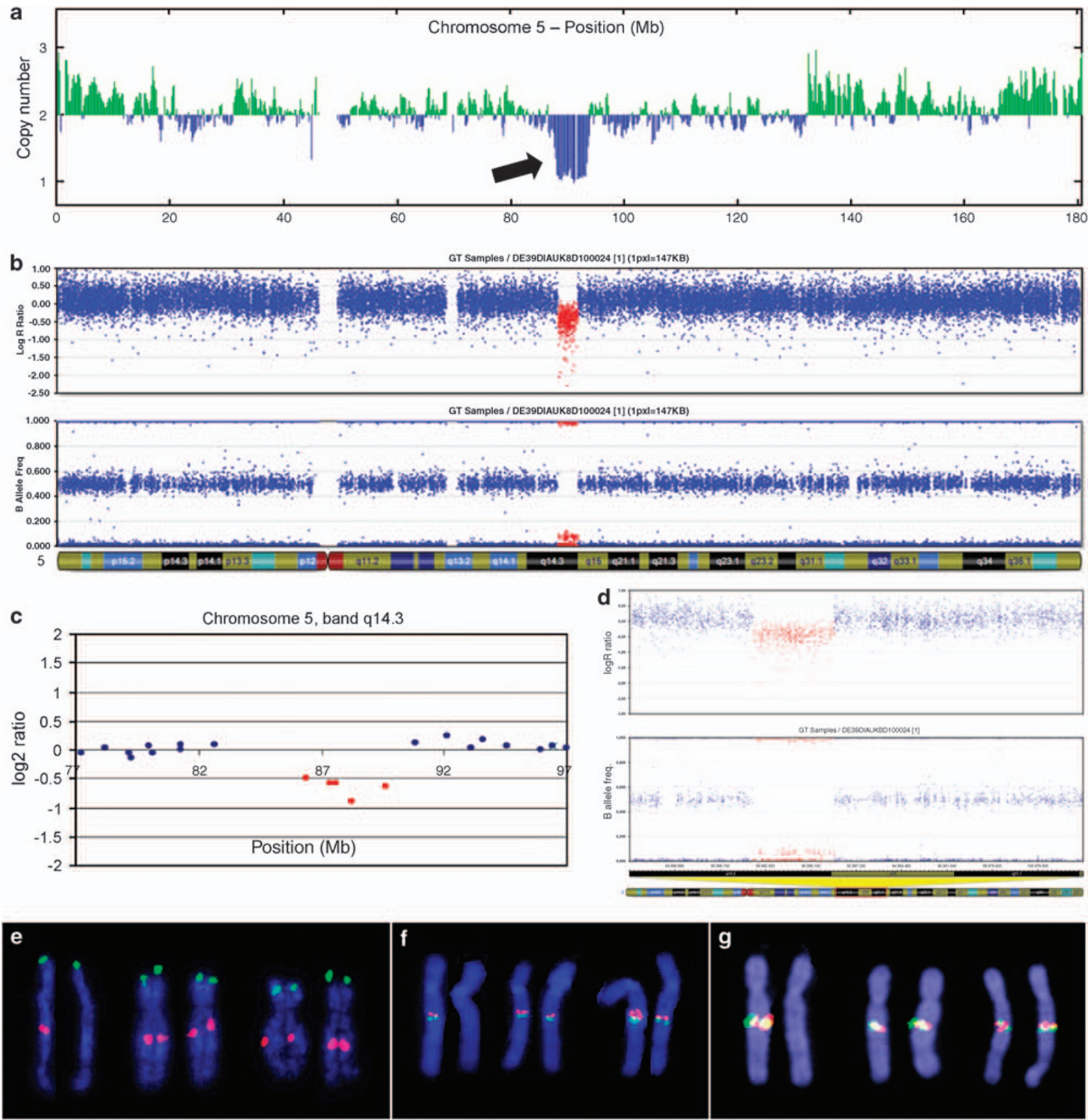

Figure 2 Summary of the molecular karyotyping and FISH results in patients 1, 2, and 3. (a) Patient 1: Affymetrix GeneChip 100K Mapping SNP array copy number plot of the entire chromosome 5 showing the $5.6 \mathrm{Mb}$ deletion in 5q14.3-q15 containing 234 SNPs. (b) Patient 3: Illumina HumanHap 550 BeadChip hybridisation plot of chromosome 5 with a $3.6 \mathrm{Mb}$ deletion involving $5 q 14.3-\mathrm{q} 15$ and containing 524 SNPs. (c) Patient 2: Partial chromosome 5 plot (Sanger $1 \mathrm{Mb}$ array) of the deletion in 5q14.3 containing 5 clones. (d) Patient 3: Partial chromosome 5 plot of the deletion 5q14.3-q15 and containing 524 SNPs. (e) Patient 1: Representative FISH results of clone RP11-88D22 (red) and subtelomeric control clone (green) in the patient (left), her mother (middle) and her father (right). (f) Patient 2: Representative FISH results of clones RP11-291O24 (red) and RP11-62E10 (green) in the patient (left), her mother (middle) and her father (right). (g) Patient 3: Representative FISH results of clones RP11-12D3 (red) and RP11-29K14 (green) in the patient (left), her mother (middle) and her father (right). In all cases, the deletions were confirmed by FISH and found to be of de novo origin.

Commonly deleted region in patients $1-3$

The region of overlap of all three deletions encompasses $1.64 \mathrm{Mb}$ in $5 \mathrm{q} 14.3$ (from genomic position $88.45 \mathrm{Mb}$ to $90.09 \mathrm{Mb})$. It contains five genes $(C E T N 3$,
AC093510.2, POLR3G, LYSMD3 and the proximal part of GPR98/MASS1) and a pseudogene (Figure 3). There were no gene interruptions at any of the six breakpoints. 
chr. 5
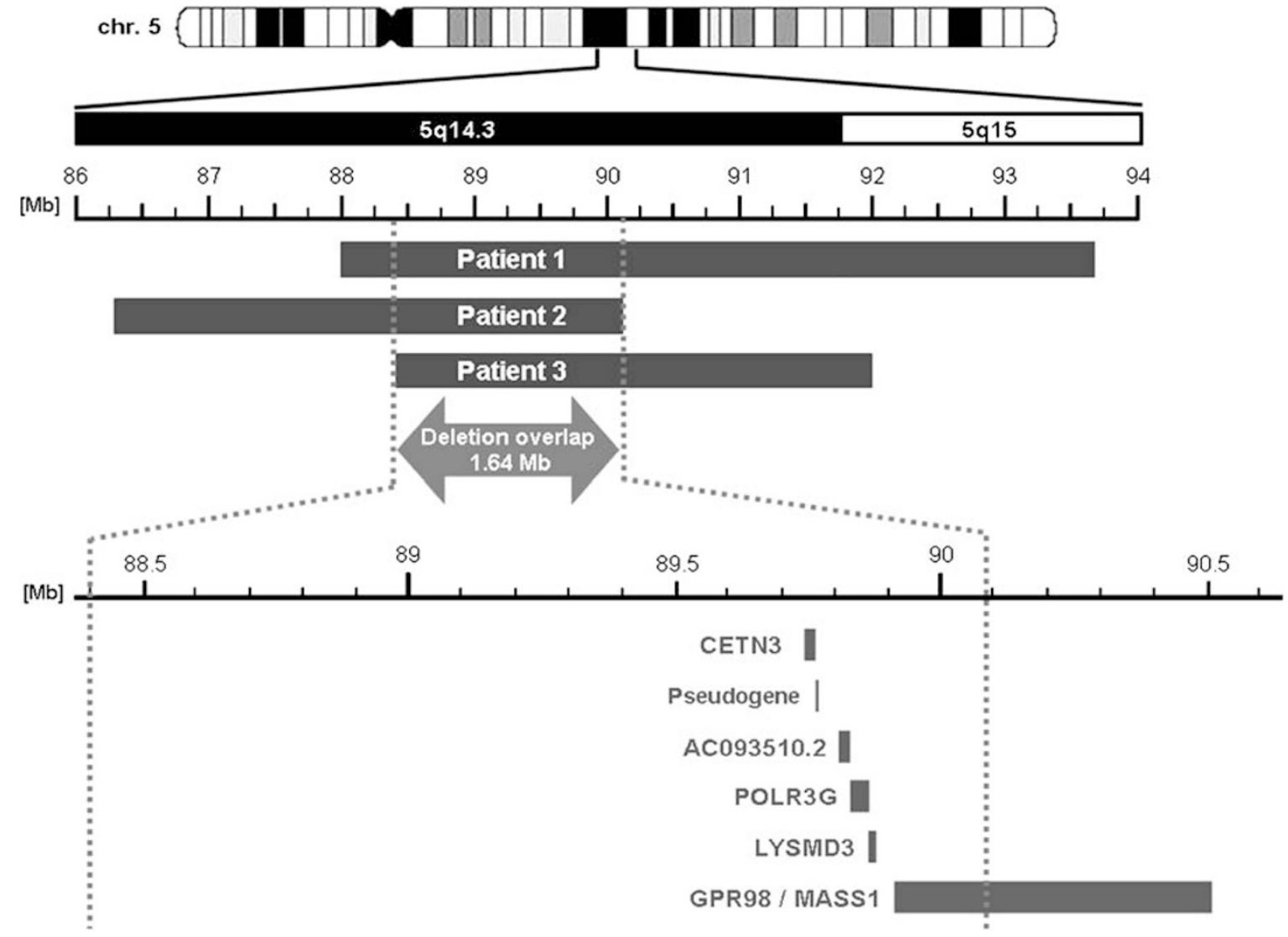

Figure 3 Schematic representation of the identified deletions involving 5q14.3-q15 in patients 1-3 (middle) and the genomic region of overlap with its gene content (bottom).

\section{Discussion}

We describe a new microdeletion syndrome caused by microdeletions within chromosomal bands 5q14.3-q15, which overlap in 5q14.3. All three patients display a common phenotype comprised of severe MR with an absence of speech and walking abilities, muscular hypotonia, seizure disorders and variable brain anomalies (Table 1). There was no consistent or striking facial dysmorphism; but mild anomalies.

The region commonly deleted in all three patients within $5 \mathrm{q} 14.3$ is approximately $1.64 \mathrm{Mb}$ in size. It contains only five genes: CETN3 (Centrin-3), AC093510.2, POLR3G (polymerase (RNA) III (DNA directed) polypeptide G), LYSMD3 (LysM, putative peptidoglycan-binding, domain containing 3) and the proximal part of GPR98/MASS1 (G-protein coupled receptor 98 precursor/monogenic audiogenic seizure susceptibility protein 1 homologue). The proximal $1.28 \mathrm{Mb}$ of the common deleted region contain no known genes. However, they contain several regions with medium to high conservation, which may be, for example, regulatory elements of adjacent, non-deleted genes and thus may play an aetiological role. Three of the genes of the common deleted region have an unspecific (POLR3G, CETN3) or as yet unknown (AC093510.2) expression pattern. POLR3G and CETN3 are expressed only weakly in murine brain ${ }^{19}$ However, two of the genes in the minimally deleted region are good candidates for the CNS phenotype of the $5 \mathrm{q} 14.3$ microdeletion syndrome patients. GPR98/Mass1 is highly expressed in the developing CNS of zebrafish embryos, particularly in the hypothalamus, epiphysis and the rhombic lips. ${ }^{20}$ Mouse embryos show a high level of expression restricted to the eyes and the developing CNS, particularly in the ventricular zone, home of neural progenitor cells during embryonal neurogenesis. ${ }^{21}$ GPR98/MASS1, a known epilepsy gene, contains both the epilepsy-associated repeat (EAR) and the epitempin (EPTP) repeat. $^{22}$ GPR98/MASS1 knockout mice show audiogenic seizure susceptibility. ${ }^{23}$ Loss of function mutations of GPR98/MASS1 have been demonstrated in an autosomal-dominant family with familial febrile seizures. ${ }^{24}$ All affected family members had normal mental and motor development and a brain MRI showed no abnormal findings.

LysmD3 is a potential single-pass membrane glycoprotein containing a LysM domain, which has been proposed as being a general peptidoglycan-binding module. LysmD3 shows an expression pattern concordant with CNS phenotypes. In early zebrafish embryos, it is expressed in the notochord, which serves both as the major skeletal 
Table 1 Phenotype of patients 1, 2 and 3 carrying microdeletions of $5 q 14.3-q 15$

\begin{tabular}{|c|c|c|c|}
\hline Features & Patient 1 & Patient 2 & Patient 3 \\
\hline Severe mental retardation & + & + & + \\
\hline $\begin{array}{l}\text { Muscular hypotonia with } \\
\text { feeding difficulties }\end{array}$ & + & + & + \\
\hline (Febrile) seizures & + & + & + \\
\hline Cranial MRI findings & $\begin{array}{l}\text { Aplasia of cerebellar vermis } \\
\text { and posterior corpus callosum, } \\
\text { multiple plexus cysts }\end{array}$ & $\begin{array}{l}\text { Prominence of the } \\
\text { arachnoid spaces in the } \\
\text { perivascular areas }\end{array}$ & $\begin{array}{l}\text { Atrophy of the supra- and infratentorial } \\
\text { region, mildly enlarged ventricular region, } \\
\text { unspecific leucoencephalopathy }\end{array}$ \\
\hline Optic atrophy & + & - & - \\
\hline $\begin{array}{l}\text { Hyperopia and strabismus } \\
\text { convergens }\end{array}$ & - & - & + \\
\hline Myocardial hypertrophy & + & - & - \\
\hline Transverse palmar creases & + & - & - \\
\hline Long fingers & + & - & + \\
\hline Café-au-lait spots & + & - & - \\
\hline Brachycephaly & - & + & - \\
\hline Low anterior hairline & - & + & - \\
\hline Facial dysmorphism & - & mild & mild \\
\hline Large ears & + & - & + \\
\hline $\begin{array}{l}\text { Visual preoccupation with } \\
\text { stripes }\end{array}$ & - & + & - \\
\hline Sensitivity to noise & - & - & + \\
\hline Frequent infections & + & - & - \\
\hline Chronic constipation & + & - & - \\
\hline $\begin{array}{l}\text { Foetal increased nuchal } \\
\text { translucency }\end{array}$ & n.n. & + & n.n. \\
\hline
\end{tabular}

n.n.: not noted.

element of the embryo and as a signalling source for the pattern formation within the neurectoderm, the paraxial mesoderm and other tissues. In later stages, high level expression of LysmD3 seems to be restricted to the CNS ${ }^{25-27}$ However, none of the four members of the LysmD family have been implied in human disease and no data from knockout models are available.

Haploinsufficiency of GPR98/MASS1 is the probable cause for the seizure phenotype in the 5q14.3 microdeletion syndrome patients presented here, because loss of function mutations have already been shown to cause seizures in humans. ${ }^{24}$ However, the carriers of these mutations had a normal psychomotor development and no abnormal findings on brain MRI. It may be hypothesized that haploinsufficiency of other genes within the de novo deletions is the cause for the additional CNS symptoms of the microdeletion patients, such as mental retardation and variable structural brain anomalies. Given its embryonic pattern of expression in animal models, LYSMD3 may be one of those haploinsufficient genes.

MEF2C is deleted in patients 1 and 2 only. It is also expressed in the brain, especially the cerebral cortex, ${ }^{19}$ and is involved in myogenesis, which may explain the more severe hypotonia and retardation observed in these two patients. Overexpression of Mef2c in mouse cardiomyocytes has been observed to cause cardiomyopathy. ${ }^{28}$ Interestingly, patient 1 presents with concentric myocardial hypertrophy and one copy of MEF2C is deleted. The unusual MRI anomalies found in the latter patient might be related to the additional deletion of NR2F1 (nuclear receptor subfamily 2, group F, member 1), which has been shown to be involved in CNS development ${ }^{29}$ and is strongly expressed in the murine cerebral cortex. ${ }^{19}$ Patient 2 also displays RASA1 deletion. Loss of function mutations in this gene, which is also expressed in brain, are related to capillary and arteriovenous malformation ${ }^{30}$ and deletion of this gene may underlie her philtral haemangioma (Figure 1g).

Our findings demonstrate a novel genomic disorder characterized by microdeletions encompassing a shortest region of overlap of $1.6 \mathrm{Mb}$ within $5 \mathrm{q} 14.3$ and a common clinical phenotype with severe MR, muscular hypotonia, seizures and variable brain and minor anomalies. Haploinsufficiency of one or more of the five genes contained in the commonly deleted region is proposed as the cause of the phenotype. GPR98/MASS1 and LYSMD3 in particular are candidate genes for seizures, MR and other CNS symptoms. This report also illustrates the power of DECIPHER as well as of national networks, such as the 'German Mental Retardation Network' (MRNET), in enabling clinical geneticists and molecular cytogeneticists from different departments to share information about rare patients to define critical intervals and evaluate their gene content and thus determine the phenotypic consequences of genomic array findings.

\section{Acknowledgements}

We thank the patients and their families for their kind cooperation. $H E, A R, D W$ and UM are members of the 'German Mental Retardation 
Network' (MRNET) which is funded by the German Federal Ministry of Education and Research (BMBF) as a part of the National Genome Research Network (NGFNplus/www.ngfn.de/englisch/15.htm, project reference numbers 01GS08164, 01GS08160). HE was also supported by the BONFOR programme of the Medical Faculty of Rheinische Friedrich-Wilhelms-University, Bonn, Grant no. O-249.0004, and LW and HVF were supported by the Cambridge Biomedical Research centre. Data for this article were retrieved from the Zebrafish Information Network (ZFIN), University of Oregon, Eugene, OR 97403-5274; http://zfin.org/ and the Allen Mouse Brain Atlas; http://www.brain-map.org.

\section{References}

1 Menten B, Maas N, Thienpont B et al: Emerging patterns of cryptic chromosomal imbalance in patients with idiopathic mental retardation and multiple congenital anomalies: a new series of 140 patients and review of published reports. J Med Genet 2006; 43: 625-633.

2 Engels H, Brockschmidt A, Hoischen A et al: DNA microarray analysis identifies candidate regions and genes in unexplained mental retardation. Neurology 2007; 68: 743-750.

3 Hoyer J, Dreweke A, Becker C et al: Molecular karyotyping in patients with mental retardation using $100 \mathrm{~K}$ single-nucleotide polymorphism arrays. J Med Genet 2007; 44: 629-636.

4 Redon R, Ishikawa S, Fitch KR et al: Global variation in copy number in the human genome. Nature 2006; 444: 444-454.

5 Mefford HC, Sharp AJ, Baker C et al: Recurrent rearrangements of chromosome 1q21.1 and variable pediatric phenotypes. N Engl J Med 2008; 359: 1685-1699.

6 Uhrig S, Schlembach D, Waldispuehl-Geigl J et al: Impact of array comparative genomic hybridization-derived information on genetic counseling demonstrated by prenatal diagnosis of the TAR (thrombocytopenia-absent-radius) syndrome-associated microdeletion 1q21.1. Am J Hum Genet 2007; 81: 866-868.

7 Shaffer LG, Theisen A, Bejjani BA et al: The discovery of microdeletion syndromes in the post-genomic era: review of the methodology and characterization of a new 1q41q42 microdeletion syndrome. Genet Med 2007; 9: 607-616.

8 Rajcan-Separovic E, Harvard C, Liu X et al: Clinical and molecular cytogenetic characterisation of a newly recognised microdeletion syndrome involving 2p15-16.1. J Med Genet 2007; 44: 269-276.

9 Hannes FD, Sharp AJ, Mefford HC et al: Recurrent reciprocal deletions and duplications of 16p13.11: The deletion is a risk factor for MR/MCA while the duplication may be a rare benign variant. J Med Genet 2009; 46: 223-232.

10 Ullmann R, Turner G, Kirchhoff $\mathrm{M}$ et al: Array CGH identifies reciprocal 16p13.1 duplications and deletions that predispose to autism and/or mental retardation. Hum Mutat 2007; 28: 674-682.

11 Brockschmidt A, Todt U, Ryu S et al: Severe mental retardation with breathing abnormalities (Pitt-Hopkins syndrome) is caused by haploinsufficiency of the neuronal bHLH transcription factor TCF4. Hum Mol Genet 2007; 16: 1488-1494.

12 Zweier C, Peippo MM, Hoyer J et al: Haploinsufficiency of TCF4 causes syndromal mental retardation with intermittent hyperventilation (Pitt-Hopkins syndrome). Am J Hum Genet 2007; 80: 994-1001.
13 Amiel J, Rio M, de Pontual L et al: Mutations in TCF4, encoding a class I basic helix-loop-helix transcription factor, are responsible for Pitt-Hopkins syndrome, a severe epileptic encephalopathy associated with autonomic dysfunction. Am J Hum Genet 2007; 80: $988-993$.

14 Koolen DA, Vissers LE, Pfundt R et al: A new chromosome $17 \mathrm{q} 21.31$ microdeletion syndrome associated with a common inversion polymorphism. Nat Genet 2006; 38: 999-1001.

15 Shaw-Smith C, Pittman AM, Willatt L et al: Microdeletion encompassing MAPT at chromosome $17 \mathrm{q} 21.3$ is associated with developmental delay and learning disability. Nat Genet 2006; 38: $1032-1037$.

16 Sharp AJ, Hansen S, Selzer RR et al: Discovery of previously unidentified genomic disorders from the duplication architecture of the human genome. Nat Genet 2006; 38: 1038-1042.

17 Shaw-Smith C, Redon R, Rickman L et al: Microarray based comparative genomic hybridisation (array-CGH) detects submicroscopic chromosomal deletions and duplications in patients with learning disability/mental retardation and dysmorphic features. J Med Genet 2004; 41: 241-248.

18 Colella S, Yau C, Taylor JM et al: QuantiSNP: an Objective Bayes Hidden-Markov Model to detect and accurately map copy number variation using SNP genotyping data. Nucleic Acids Res 2007; 35: 2013-2025.

19 Lein ES, Hawrylycz MJ, Ao N et al: Genome-wide atlas of gene expression in the adult mouse brain. Nature 2007; 445: 168-176.

20 Gibert Y, McMillan DR, Kayes-Wandover K, Meyer A, Begemann G, White PC: Analysis of the very large G-protein coupled receptor gene (Vlgr1/Mass1/USH2C) in zebrafish. Gene 2005; 353: 200-206.

21 McMillan DR, Kayes-Wandover KM, Richardson JA, White PC: Very large $\mathrm{G}$ protein-coupled receptor-1, the largest known cell surface protein, is highly expressed in the developing central nervous system. J Biol Chem 2002; 277: 785-792.

22 Robinson R, Gardiner M: Molecular basis of Mendelian idiopathic epilepsies. Ann Med 2004; 36: 89-97.

23 Yagi $\mathrm{H}$, Takamura Y, Yoneda $\mathrm{T}$ et al: Vlgr1 knockout mice show audiogenic seizure susceptibility. J Neurochem 2005; 92: 191-202.

24 Nakayama J, Fu YH, Clark AM et al: A nonsense mutation of the MASS1 gene in a family with febrile and afebrile seizures. Ann Neurol 2002; 52: 654-657.

25 Stemple DL, Solnica-Krezel L, Zwartkruis F et al: Mutations affecting development of the notochord in zebrafish. Development 1996; 123: 117-128.

26 Thisse B, Heyer V, Lux A et al: Spatial and temporal expression of the zebrafish genome by large-scale in situ hybridization screening. Methods Cell Biol 2004; 77: 505-519.

27 Sprague J, Bayraktaroglu L, Bradford Y et al: The Zebrafish Information Network: the zebrafish model organism database provides expanded support for genotypes and phenotypes. Nucleic Acids Res 2008; 36: D768-D772.

$28 \mathrm{Xu} \mathrm{J}$, Gong NL, Bodi I, Aronow BJ, Backx PH, Molkentin JD: Myocyte enhancer factors $2 \mathrm{~A}$ and $2 \mathrm{C}$ induce dilated cardiomyopathy in transgenic mice. J Biol Chem 2006; 281: 9152-9162.

29 Naka H, Nakamura S, Shimazaki T, Okano H: Requirement for COUP-TFI and II in the temporal specification of neural stem cells in CNS development. Nat Neurosci 2008; 11: 1014-1023.

30 Eerola I, Boon LM, Mulliken JB et al: Capillary malformationarteriovenous malformation, a new clinical and genetic disorder caused by RASA1 mutations. Am J Hum Genet 2003; 73: 1240-1249. 